\title{
Topological phases and edge states in a non-Hermitian trimerized optical lattice
}

\author{
L. Jin ${ }^{*}$ \\ School of Physics, Nankai University, Tianjin 300071, China
}

(Received 1 July 2017; published 5 September 2017)

\begin{abstract}
Topologically engineered optical materials support robust light transport. Herein, the investigated nonHermitian lattice is trimerized and inhomogeneously coupled using uniform intracell coupling. The topological properties of the coupled waveguide lattice are evaluated and we find that the $\mathcal{P} \mathcal{T}$-symmetric phase of a $\mathcal{P} \mathcal{T}$-symmetric lattice can have different topologies; the edge states depend on the lattice size, boundary configuration, and competition between the coupling and degree of non-Hermiticity. The topologically nontrivial region is extended in the presence of periodic gain and loss. The nonzero geometric phases accumulated by the Bloch bands indicate the existence of topologically protected edge states between the band gaps. The unidirectional amplification and attenuation zero modes appear above a threshold degree of non-Hermiticity, which facilitates the development of a robust optical diode.
\end{abstract}

DOI: 10.1103/PhysRevA.96.032103

\section{INTRODUCTION}

Topological insulators are novel states of solid-state materials that have an insulating bulk band gap and a conducting edge or surface [1]. The edge or surface states are symmetry protected against local disorder and valuable for quantum transport and computation in quantum (anomalous) Hall insulators and quantum spin Hall insulators [2]. The optical realization of topological systems has stimulated the field of topological photonics, which enables the experimental studies of topological systems that are difficult to realize in condensed-matter physics. The photons in coupled waveguides and optical lattices are manipulated in a manner similar to the electrons in solids, providing intriguing opportunities for novel optical devices [3-5]. The topologically protected unidirectional interface state propagates robustly against local impurities. This was experimentally demonstrated in coupled waveguide ring resonators [6]. Synthesizing artificial gauge fields for ultracold atoms in optical lattices enables the construction of a two-dimensional topological system [7]. The Su-Schrieffer-Heeger (SSH) model [8] is the simplest system that has topologically nontrivial edge states [9-20]; this model has been realized for a dimerized optical superlattice, wherein the topological properties of Bloch bands were experimentally measured [21].

As progress on topological photonics has advanced, tremendous interest has also been paid to parity-time $(\mathcal{P} \mathcal{T})$ symmetric non-Hermitian systems in coupled optical waveguides [22-25], resonators [26-32], atoms, and atomic lattices $[33,34] . \mathcal{P} \mathcal{T}$-symmetric systems can have an entirely real spectrum, although they are non-Hermitian [35-43]. Intriguing phenomena have been revealed including fast evolution [44-47], power oscillation [48], and unidirectional reflectionless [49] and coherent absorption [50-52]. The $\mathcal{P} \mathcal{T}$-symmetric properties of an $\mathrm{SSH}$ chain with a pair of $\mathcal{P} \mathcal{T}$-symmetric

\footnotetext{
*jinliang@nankai.edu.cn

Published by the American Physical Society under the terms of the Creative Commons Attribution 4.0 International license. Further distribution of this work must maintain attribution to the author(s) and the published article's title, journal citation, and DOI.
}

potentials at boundaries have been studied [53-55]; the $\mathcal{P} \mathcal{T}$ transition threshold has a power-law decay with the SSH chain size [54]; the Hilbert space inner product has been constructed in the framework of pseudo-Hermitian quantum mechanics, which provides deep insight into and novel physics on the non-Hermitian topological systems [55]. Moreover, the edge states are unaffected by the gain and loss when zero probabilities are located at the edges [56,57].

Because extensive progress has been made in topological photonics and $\mathcal{P} \mathcal{T}$-symmetric optics, the optical analysis of topological systems has been extended to non-Hermitian systems [58-61]. The topological interface states in nonHermitian systems have been systematically discussed [62]. Topologically protected $\mathcal{P} \mathcal{T}$-symmetric interface states were demonstrated in coupled resonators [63]. Robust light interface states were discovered at the interface between SSH chains with distinct non-Hermiticity [64]. Topological properties are characterized using the generalized winding number in non-Hermitian systems [65]. $\mathcal{P} \mathcal{T}$-symmetric non-Hermitian Aubry-André systems [66,67] and Kitaev models [68,69] were theoretically investigated. Evidence reveals that universal nonHermiticity may alter topological regions [70], but topological properties are robust against local non-Hermiticity.

In this paper, we investigate a coupled waveguide lattice that has a trimerized unit cell and in which gain and loss are balanced and separated by a passive waveguide. The lattice has universal non-Hermiticity. The intracell coupling $g_{1}$ is uniform and differs from the intercell coupling $g_{2}$. The nontrivial topology of the lattice implies that it has a conducting edge or surface. Under the periodic boundary condition, the geometric phases accumulated are $\pi$ when the upper and lower bands circle one loop in the Brillouin zone in the topologically nontrivial region; corresponding edge states exist between the band gaps under an open boundary condition. In $\mathcal{P} \mathcal{T}$-symmetric configurations, the $\mathcal{P} \mathcal{T}$-symmetric regions possess different topologies depending on the coupling configuration and universal non-Hermiticity. In particular, we discover a non-Hermitian threshold above which there exist unidirectional amplified and damped edge states, which are located at opposite boundaries with identical resonant frequency. The topologically nontrivial regions with edge states are widened because of the non-Hermitian periodic 

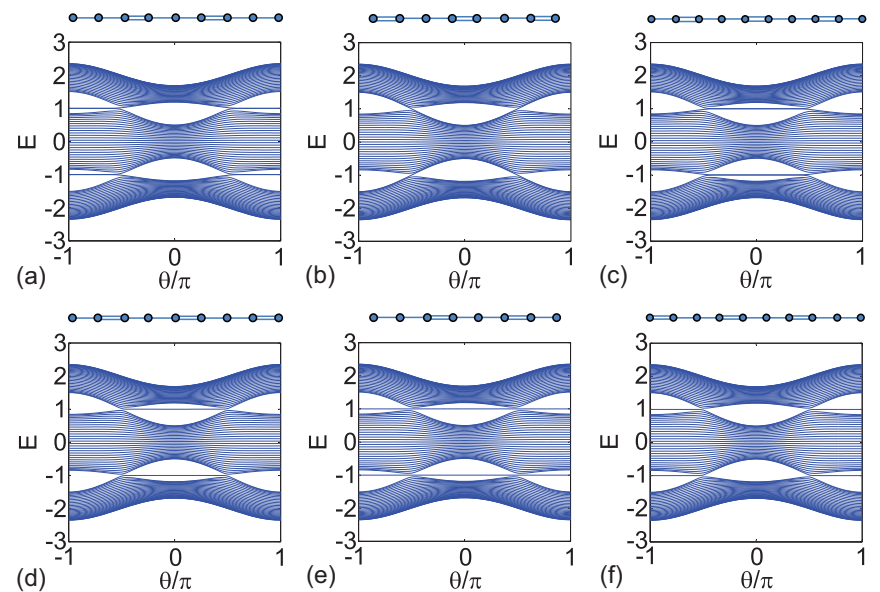

FIG. 1. Energy bands for (a)-(c) $\mathcal{P} \mathcal{T}$-symmetric and (d)-(f) non$\mathcal{P} \mathcal{T}$-symmetric configurations for (a) and (d) $N=90$, (b) and (e) $N=89$, and (c) and (f) $N=88$. Lattice configurations are displayed above energy bands. Here $\Delta=1 / 2$.

gain and loss. The threshold corresponds to a bifurcation point in the imaginary part of the spectrum. The topological region expands when the lattice has substantial non-Hermiticity.

The reminder of the paper is organized as follows. In Sec. II we discuss the topological properties of a Hermitian trimerized lattice. In Secs. III and IV we investigate the lattice's $\mathcal{P} \mathcal{T}$-symmetric non-Hermitian extension based on coupled waveguides. The band structure, $\mathcal{P} \mathcal{T}$-symmetric phases, and edge states are investigated. The results are summarized in Sec. V.

\section{THE TRIMERIZED LATTICE}

We focus on the topological properties of a trimerized lattice, which has more edge states than a dimerized $\mathrm{SSH}$ chain [53-55]. The topologically protected edge states are related to the lattice structure and are determined by the boundary $[71,72]$. The trimerized lattice investigated herein comprises three groups, which are indicated by lattice number $N=3 n, 3 n-1$, and $3 n-2$; when considering the coupling configurations, the lattice is divided into three groups by their inhomogeneous couplings at the boundary: $g_{1}-g_{1}-g_{2^{-}}$, $g_{2}-g_{1^{-}} g_{1^{-}}$, and $g_{1^{-}} g_{2^{-}}-g_{1^{-}}$. The lattice has only six topologically distinct configurations because of its reflection symmetry. These configurations are illustrated above their spectra presented in Fig. 1, where each site represents a waveguide. The three configurations in Figs. 1(a)-1(c) are $\mathcal{P} \mathcal{T}$ symmetric, whereas those in Figs. 1(d)-1(f) are not. The differences in the configuration of the trimerized lattice result in more edge states in the topologically nontrivial phase. The trimerized lattice that we consider can be modeled using a one-dimensional offdiagonal Aubry-André-Harper (AAH) Hamiltonian $H_{\mathrm{AAH}}=$ $\sum_{j}\left[1+\lambda \cos \left(2 \pi \beta j+\phi_{\lambda}\right)\right] a_{j}^{\dagger} a_{j+1}+$ H.c., where $a_{j}^{\dagger}\left(a_{j}\right)$ is the creation (annihilation) operator for bosonic particles. A rational number of $\beta=1 / 3$ leads to a trimerized lattice with three bands. The amplitude $\lambda$ and phase factor $\phi_{\lambda}$ control the modulation of coupling strength. At $\phi_{\lambda}=2 m \pi / 3$ (where $m$ is an integer), the three repeated couplings are $\left\{g_{1}, g_{1}, g_{2}\right\}$ with $g_{1}=1-\lambda / 2$ and $g_{2}=1+\lambda$.
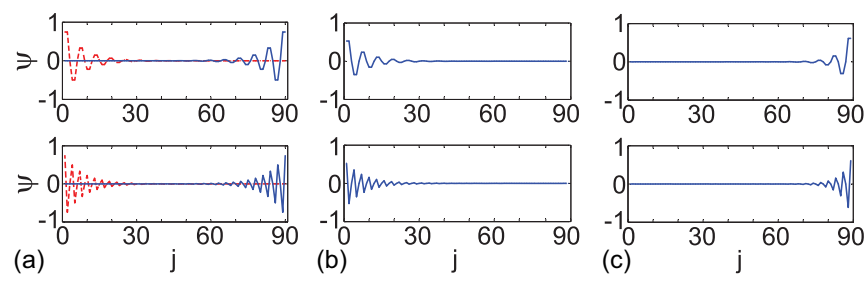

FIG. 2. Three types of edge states for configurations in (a) Fig. 1(a) at $\theta=\pi$, (b) Fig. 1(e) at $\theta=\pi$, and (c) Fig. 1(e) at $\theta=0$. The edge states in Fig. 1(c) at $\theta=0$ are depicted in (a), those in Fig. 1(d) at $\theta=0$ are depicted in (b), and those in Fig. 1(f) at $\theta=\pi$ are depicted in (c). Here $\Delta=1 / 2$.

To investigate the topological properties of the trimerized lattice, we set the intracell coupling $g_{1}$ to unity and force the intercell coupling to change periodically as

$$
g_{2}=1-\Delta \cos \theta
$$

The energy bands are plotted as a function of $\theta$ in Fig. 1 . The single line indicates the intracell coupling $g_{1}$ and the double lines indicate the intercell coupling $g_{2}$. Edge states with energies \pm 1 exist between the upper and lower band gaps and are symmetrically arranged about zero energy. In Fig. 1(a), four edge states (twofold degenerate) are present in the regions $-\pi<\theta<-\pi / 2$ and $\pi / 2<\theta<\pi$. The four edge states are displayed in Fig. 2(a), wherein the upper panel shows two degenerate edge states of energy +1 that are localized on the left and right boundaries, respectively. The amplitudes are approximately $\{1,1,0\}$ for every three sites from the boundaries. The lower panel shows the two edge states with energy -1 ; the corresponding amplitudes are approximately $\{1,-1,0\}$ for every three sites from the boundaries. In Fig. 1(b), the couplings at the left and right chain boundaries are the intercell coupling $g_{2}$ and there is no edge state in any region of $\theta$. In Fig. 1(c), four edge states (twofold degenerate) exist in the region $-\pi / 2<\theta<\pi / 2$. In Fig. 1(d), there are two edge states localized on the left boundary in the region $-\pi / 2<\theta<\pi / 2$, as shown in Fig. 2(b), but no edge states are localized on the right boundary. In Fig. 1(e), there are also two edge states, in the region $-\pi / 2<\theta<\pi / 2$, and they are both localized on the right boundary [Fig. 2(c)]. In other regions $(-\pi<\theta<-\pi / 2$ and $\pi / 2<\theta<\pi)$, the edge states are localized on the left boundary [Fig. 2(b)]. In Fig. 1(f), two edge states exist in the regions $-\pi<\theta<-\pi / 2$ and $\pi / 2<\theta<\pi$, localized on the right boundary.

The existence of edge states is related to the lattice configuration. Edge states exist when the coupling $g_{1}$ is experienced at the lattice boundary. The configuration with $g_{1}-g_{2}-g_{1}$ - at the boundary results in two edge states of energy \pm 1 in the region $-\pi / 2<\theta<\pi / 2$, whereas the configuration with $g_{1}-g_{1}-g_{2}$ - results in two edge states of energy \pm 1 in the regions $-\pi<\theta<-\pi / 2$ and $\pi / 2<\theta<\pi$. When the lattice has reflection symmetry, there are four edge states (twofold degenerate). The edge states disappear in the configuration with $g_{2}-g_{1}-g_{1}$ - at the boundary. The edges states appear at three cases. The edge states plotted in Fig. 2(a) are for the lattice with reflection symmetry [Fig. 1(a)]. Twofold degenerate edge states are located at the left and right boundaries, respectively. In Figs. 2(b) and 2(c), the edge states for the configuration 
presented in Fig. 1(e) are plotted; in this situation, one pair of edge states with energy \pm 1 is located on one boundary. The edge state amplitude is approximately $\{1, \pm 1,0\}$ for every three sites from the boundary. The amplitude decays according to $\left(g_{1} / g_{2}\right)^{p}$ in the regions $-\pi<\theta<-\pi / 2$ and $\pi / 2<\theta<\pi$ and according to $\left(g_{2} / g_{1}\right)^{p}$ in the region $-\pi / 2<\theta<\pi / 2$ from the lattice boundary (where $p$ is the unit cell index). Inside the unit cell, the nonzero amplitudes of edge states are symmetric (upper panel in Fig. 2) and antisymmetric (lower panel in Fig. 2). For edge state with energy +1 , the corresponding amplitude has overall phase difference $e^{i \pi}$ between neighbor unit cells; for the edge state with energy -1 , the amplitude has no such phase difference, as illustrated in Fig. 2.

\section{THE $\mathcal{P} \mathcal{T}$-SYMMETRIC NON-HERMITIAN LATTICE}

In this section, we investigate a one-dimensional trimerized lattice of evanescently coupled optical waveguides. The lattice has three sublattices $A, B$, and $C$ and this spatial arrangement induces inhomogeneous couplings. The spacing between waveguides $A$ and $B$ is equal to that between $B$ and $C$ in a unit cell, which differs from the spacing between waveguides $A$ and its nearest neighbor $C$. This spatial arrangement induces a periodic modulation in every the third coupling. The amplitudes of the waveguides in a unit cell are denoted by $\psi_{m, A}, \psi_{m, B}$, and $\psi_{m, C}$, where $m$ labels the unit cell index. In coupled-mode theory, the single-mode-coupled waveguide lattice is modeled by a tight-binding system as follows:

$$
\begin{gathered}
i \dot{\psi}_{m, A}=-i \gamma_{A} \psi_{m, A}+g_{2} \psi_{m-1, C}+g_{1} \psi_{m, B}, \\
i \dot{\psi}_{m, B}=-i \gamma_{B} \psi_{m, B}+g_{1} \psi_{m, A}+g_{1} \psi_{m, C}, \\
i \dot{\psi}_{m, C}=-i \gamma_{C} \psi_{m, C}+g_{1} \psi_{m, B}+g_{2} \psi_{m+1, A},
\end{gathered}
$$

where $g_{1}$ is the intracell coupling and $g_{2}$ is the intercell coupling. The waveguides losses are denoted by $\gamma_{A}, \gamma_{B}$, and $\gamma_{C}$ for sublattices $A, B$, and $C$, respectively. Under the condition $\gamma_{B}-\gamma_{A}=\gamma_{C}-\gamma_{B} \equiv \gamma$, the system reduces to a $\mathcal{P} \mathcal{T}$-symmetric lattice with on-site potentials $\{i \gamma, 0,-i \gamma\}$ in a three-site unit cell after the removal of a common loss rate $\gamma_{B}$. The trimerized lattice chain is described by a $\mathcal{P} \mathcal{T}$-symmetric off-diagonal AAH Hamiltonian with non-Hermitian on-site potentials, i.e., $H=H_{\mathrm{AAH}}+(-2 i \gamma / \sqrt{3}) \sum_{j} \cos (2 \pi j / 3+$ $\pi / 6) a_{j}^{\dagger} a_{j}$. The Hamiltonian $H$ can be rewritten in a matrix form. Examples of the SSH models are listed in Ref. [55].

Figure 3(a) schematically plots the $\mathcal{P} \mathcal{T}$-symmetric lattice under the periodic boundary condition. For $N=3 n$ and taking the Fourier transformation of Bloch waves, the waveguide lattice matrix is expressed as $H=\sum_{k} H_{k}$, where the wave vector $k=2 \pi m / n(m=1,2, \ldots, n)$ and

$$
H_{k}=\left(\begin{array}{ccc}
i \gamma & g_{1} & g_{2} e^{i k} \\
g_{1} & 0 & g_{1} \\
g_{2} e^{-i k} & g_{1} & -i \gamma
\end{array}\right) .
$$

Notably, $H_{k}$ is $\mathcal{P} \mathcal{T}$ symmetric $\left[\mathcal{P} \mathcal{T} H_{k}(\mathcal{P} \mathcal{T})^{-1}=H_{k}\right]$ and includes three energy bands. Here $\mathcal{P}$ is defined as the parity operator and satisfies $\mathcal{P} A_{k} \mathcal{P}^{-1}=C_{k}, \mathcal{P} B_{k} \mathcal{P}^{-1}=B_{k}$, and $\mathcal{P} C_{k} \mathcal{P}^{-1}=A_{k}$ (where $A_{k}, B_{k}$, and $C_{k}$ are the corresponding sublattices in Bloch wave-vector space); $\mathcal{T}$ is defined as the
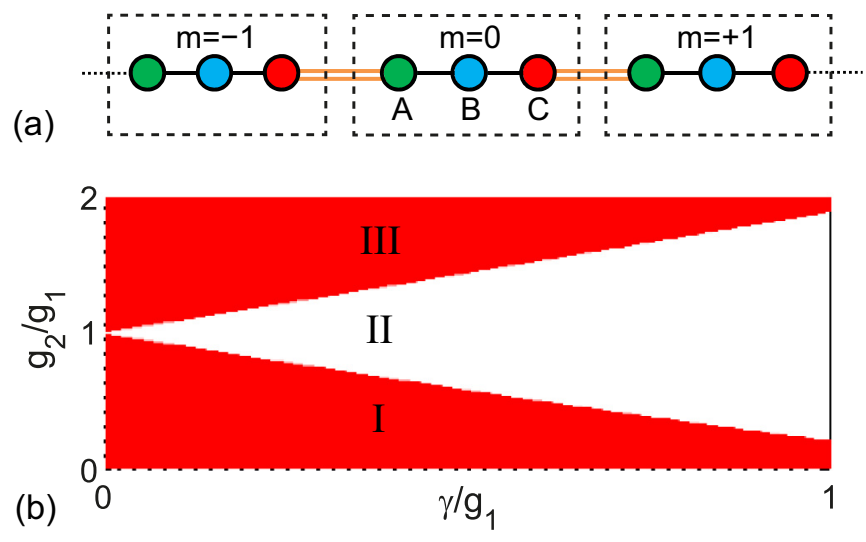

FIG. 3. (a) One-dimensional trimerized $\mathcal{P} \mathcal{T}$-symmetric lattice. The unit cell has balanced gain and loss $\{i \gamma, 0,-i \gamma\}$ in the dashed rectangles. Gain and loss are represented by green and red, respectively. The passive lattice is displayed in cyan. (b) Phase diagram in the $\gamma-g_{2}$ space. Region II is the broken- $\mathcal{P} \mathcal{T}$-symmetry phase; region I (III) is the exact $\mathcal{P} \mathcal{T}$-symmetric phase in the topologically trivial (nontrivial) region.

time-reversal operator, which satisfies $\mathcal{T} i \mathcal{T}^{-1}=-i$. The band gaps are closed at the boundaries of the Brillouin zone: $k=0$ and $\pi$. The $\mathcal{P} \mathcal{T}$ transition occurs at $\left(\gamma^{2}-2 g_{1}^{2}-g_{2}^{2}\right)^{3} / 3^{3}+$ $\left(2 g_{1}^{2} g_{2} \cos k\right)^{2} / 2^{2}=0$. The phase diagram is depicted in Fig. 3(b). Region II (white) represents the region in which $\mathcal{P} \mathcal{T}$ symmetry is broken. Regions I and III are the regions of exact $\mathcal{P} \mathcal{T}$ symmetry. Inhomogeneity is necessary for the existence of an exact $\mathcal{P} \mathcal{T}$-symmetric phase and monotonically increases with the degree of non-Hermiticity.

The lattice topology is identified by the Zak phases of the energy bands [73,74], which are defined as $\theta=$ $i \int_{-\pi}^{\pi} d k\left\langle\psi_{R, k} \mid d \psi_{L, k} / d k\right\rangle$ in a non-Hermitian system, where $\psi_{L, k}$ and $\psi_{R, k}$ are the left and right eigenstates of $H_{k}[15,64]$. The geometric phases accumulated are $\pi, 0, \pi$ for $g_{1}<g_{2}$ when each Bloch band circles one loop in the Brillouin zone and 0,0,0 for $g_{1}>g_{2}$. Bulk-edge correspondence means that edge states exist in the band gaps under an open boundary condition. The exact $\mathcal{P} \mathcal{T}$-symmetric regions I and III have topologically distinct phases. In the $\mathcal{P} \mathcal{T}$-symmetric region I, the geometric phases accumulated for the three bands are all zero, which implies that region $\mathrm{I}$ is a topologically trivial phase; in the $\mathcal{P} \mathcal{T}$-symmetric region III, the geometric phases accumulated are 0 for the middle band and $\pi$ for the upper and lower bands, which reflects that region III is a topologically nontrivial phase, and edge states exist simultaneously between the two band gaps under an open boundary condition similar to that shown in Fig. 1. As intercell coupling $g_{2}$ is varied from 0 to $2 g_{1}$, the system transitions from an exact $\mathcal{P} \mathcal{T}$-symmetric phase with trivial topology, through the broken- $\mathcal{P} \mathcal{T}$-symmetry phase, to an exact $\mathcal{P} \mathcal{T}$-symmetric phase with nontrivial topology.

\section{EDGE STATES}

The existence of a topologically protected edge state is an important feature of topological systems. In Fig. 4, the real and imaginary parts of the spectra for $N=3 n, 3 n-1$, and $3 n-2$ at different $\gamma$ are plotted. The lattice is the most sensitive to 

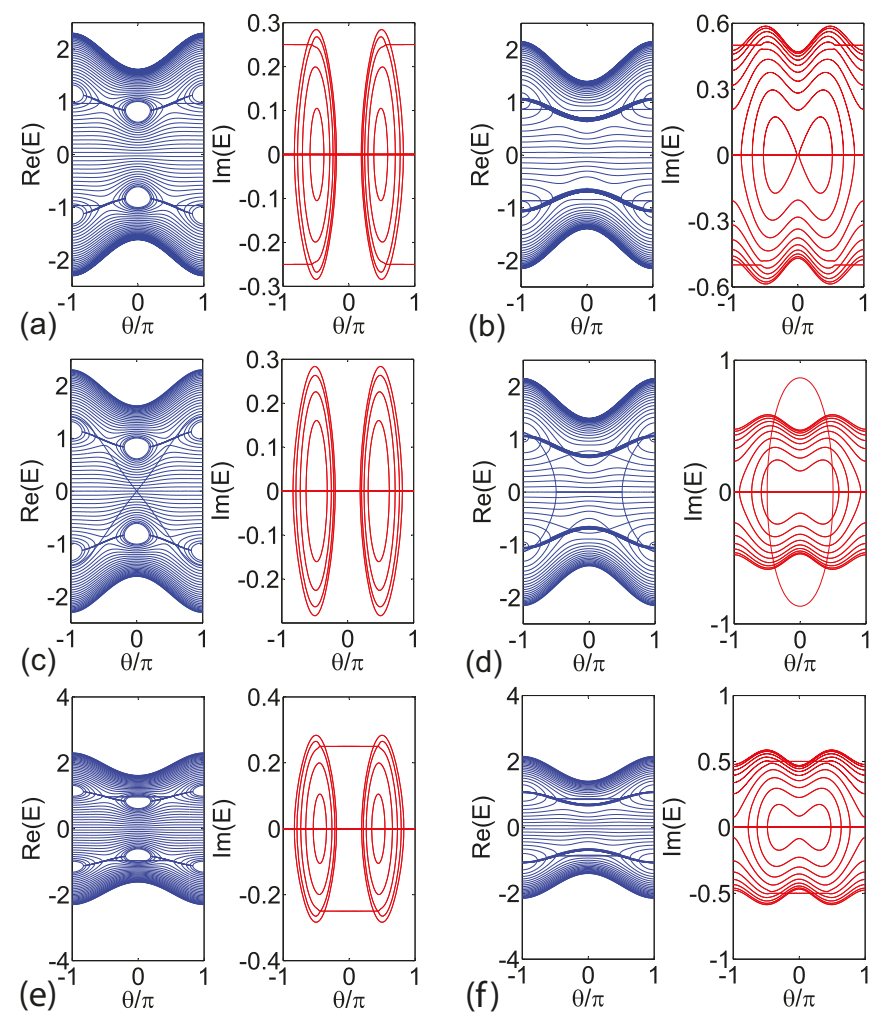

FIG. 4. Energy bands of $\mathcal{P} \mathcal{T}$-symmetric configurations for (a) and (b) $N=3 n$, (c) and (d) $N=3 n-1$, and (e) and (f) $N=3 n-2$ for (a), (c), and (e) $\gamma=1 / 2$ and (b), (d), and (f) $\gamma=1$. Here $n=30$ and $\Delta=1 / 2$.

$\mathcal{P} \mathcal{T}$-symmetric gain and loss at $g_{1}=g_{2}(\theta= \pm \pi / 2)$. The broken energy levels are raised as $\gamma$ is increased and the band gaps narrow. Edge states emerge in the bands, but their frequency and amplifying or damping rate are independent of the intercell coupling $g_{2}$ for a lattice without defects at the boundary $(N=3 n)$. For $N=3 n-1$, the topology is not affected by weak non-Hermiticity and there are no edge states [Fig. 4(c)]. However, an edge state exists at large non-Hermiticity when $\gamma>g_{2}$ [Fig. 4(d)] and its frequency is resonant with that of the waveguide; the amplifying (damping) rate is $g_{2}$ dependent and increases with $\gamma$. We calculate the edge states' resonant frequency and estimate the threshold gain and loss rate as follows.

Three $\mathcal{P} \mathcal{T}$-symmetric configurations are illustrated in Figs. 1(a)-1(c). Topologically protected edge states exist in the configurations in Figs. 1(a) and 1(c) in the absence of gain and loss when the system is Hermitian. Notably, the system topology is robust against the non-Hermiticity. In the presence of gain and loss, the Hamiltonian still commutes with the $\mathcal{P} \mathcal{T}$ operator. Consequently, the eigenvalues become real or conjugate pairs. The edge state on one side switches to the other side of the lattice chain after the $\mathcal{P} \mathcal{T}$ operation; therefore, the edge state is not the eigenstate of the $\mathcal{P} \mathcal{T}$ operator and $\mathcal{P} \mathcal{T}$ symmetry breaks. The edge states appear in conjugate pairs, which are robust amplification or attenuation modes. In the topological non-Hermitian optical lattice, the coexistence of topology and non-Hermiticity enables the lattice to function as a robust optical diode.
The Schrödinger equations for the edge states localized on the right side satisfy $\psi_{N-2}=0, E \psi_{N-1}=g_{1} \psi_{N}$, and $(E+i \gamma) \psi_{N}=g_{1} \psi_{N-1}$, from which we obtain the edge state eigenvalues

$$
E_{-, \pm}=\left(-i \gamma \pm \sqrt{4 g_{1}^{2}-\gamma^{2}}\right) / 2,
$$

which are damped modes because of the $-i \gamma / 2$ term in $E_{-, \pm}$. Indeed, the damping is because the edge state probability is mainly distributed on the lossy sites. The amplitude of the edge states decays as $\left(g_{1} / g_{2}\right)^{p}$ from the unit cell on the right boundary. The wave-function amplitude is $\left\{0, \sigma_{+, \pm}, 1\right\}$ in each unit cell, where $\sigma_{+, \pm}=\left(i \gamma \pm \sqrt{4 g_{1}^{2}-\gamma^{2}}\right) / 2 g_{1}$ indicates the wave-function distribution. The edge states can be expressed as

$$
\psi_{r}=\left[0,\left(g_{1} / g_{2}\right)^{m-1} \sigma_{+, \pm},\left(g_{1} / g_{2}\right)^{m-1}, \ldots, 0, \sigma_{+, \pm}, 1\right]^{T}
$$

for the system $N=3 m$. For edge states localized on the left side, the eigenvalues are

$$
E_{+, \pm}=\left(+i \gamma \pm \sqrt{4 g_{1}^{2}-\gamma^{2}}\right) / 2 .
$$

These two edge states are amplified modes. The wave-function distribution is

$$
\psi_{l}=\left[1, \sigma_{-, \pm}, 0, \ldots,\left(g_{1} / g_{2}\right)^{m-1},\left(g_{1} / g_{2}\right)^{m-1} \sigma_{-, \pm}, 0\right]^{T} .
$$

The decay factor is $g_{1} / g_{2}$ and the amplitude in the unit cell is $\left\{1, \sigma_{-, \pm}, 0\right\}$, where $\sigma_{-, \pm}=\left(-i \gamma \pm \sqrt{4 g_{1}^{2}-\gamma^{2}}\right) / 2 g_{1}$. The edge states are depicted in Fig. 2(a) for $\gamma=0$. When the gain and loss are introduced, the twofold degenerate edge states in each band gap become one amplified and one damped pair, with the edge state damping or amplifying rate given by $\gamma$. The frequencies of the edge states are reduced from $\pm g_{1}$ to $\pm \sqrt{g_{1}^{2}-\gamma^{2} / 4}$ in the upper and lower band gaps separated by $\sqrt{4 g_{1}^{2}-\gamma^{2}}$. The first \pm in the subscript of $E_{ \pm, \pm}$indicates that the edge states are damped or amplified; the second \pm in the subscript of $E_{ \pm, \pm}$indicates that the edge states are in the upper or lower band gap.

The edge states for $\gamma=0$ are depicted in Figs. 2(b) and 2(c). When $\gamma \neq 0$, the right-side edge states are expressed as

$$
\psi_{r}=\left[0,\left(g_{2} / g_{1}\right)^{m-1} \sigma_{-, \pm},\left(g_{2} / g_{1}\right)^{m-1}, \ldots, 0, \sigma_{-, \pm}, 1\right]^{T}
$$

and the left-side edge states are expressed as

$$
\psi_{l}=\left[1, \sigma_{+, \pm}, 0, \ldots,\left(g_{2} / g_{1}\right)^{m-1},\left(g_{2} / g_{1}\right)^{m-1} \sigma_{+, \pm}, 0\right]^{T} .
$$

The decay factor is $g_{2} / g_{1}$. Here $E_{ \pm,+}\left(E_{ \pm,-}\right)$corresponds to the edge states in the upper (lower) band gap. When $g_{2}$ is on the boundary, two edge states do not exist. If the lattice has a different boundary of $g_{1}-g_{2}-g_{1}$ - and $g_{1}-g_{1}-g_{2}$ - on the edge, the system has either right or left edge states, except when $g_{1}=g_{2}$.

Three types of $\mathcal{P} \mathcal{T}$-symmetric configurations are schematically illustrated in the $\mathcal{P} \mathcal{T}$-symmetric lattice in Fig. 5. In all configurations, the repeated unit cells are identical; the lattice numbers are distinct. The balanced gain and loss in the unit cell are $\{-i \gamma, 0, i \gamma\}$. In Figs. 5(b) and 5(c), the unit cells are defective at the edges. This leads to the existence of different edge states in the corresponding configurations. In particular, edge states exist in conjugate pairs as the 
(a)

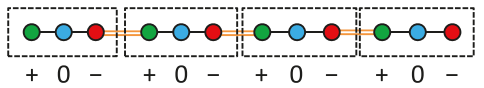

(b)

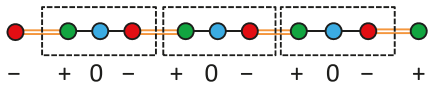

(c)

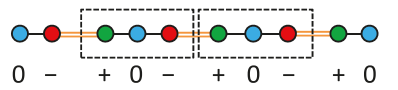

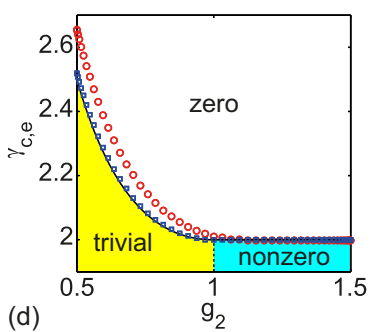

(d)

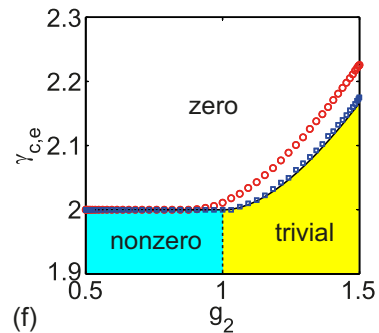

FIG. 5. $\mathcal{P} \mathcal{T}$-symmetric lattices with (a) integer unit cells $(N=3 n)$, (b) a single additional site at each boundary $(N=3 n-1)$, and (c) two additional sites at each boundary $(N=3 n-2)$. The threshold gain (loss) rate $\gamma_{\mathrm{c}, \mathrm{e}}$ for the real part of the edge state eigenvalues vanishes, as depicted for (d) $N=3 n$, (e) $N=3 n-1$, and (f) $N=3 n-2$. Red circles (blue squares) display numerical calculations for the lattice with $n=30$ ( $n=300)$; black lines present the analytical results for infinite systems $n \rightarrow \infty$. Trivial (yellow area), zero (white area), and nonzero (cyan area) in (d)-(f) indicate the topologically trivial and nontrivial regions with the real part of the edge state eigenvalues being zero and nonzero, respectively.

degree of non-Hermiticity increases, and the real parts of their eigenvalues are zero.

The broken- $\mathcal{P} \mathcal{T}$-symmetry edge states with real eigenvalues equal to zero have been demonstrated in honeycomb and square lattices [65]. In the configuration illustrated in Fig. 1(b), the chain number is $N=3 n-1$ and no edge state exists in the Hermitian system (at $\gamma=0$ ). Affected by the universal gain and loss, the edge states exist when $\gamma>g_{2}$. The Schrödinger equations for the edge state wave functions satisfy $\psi_{N-2}=$ $0,(E+i \gamma) \psi_{N-1}=g_{2} \psi_{N}$, and $(E-i \gamma) \psi_{N}=g_{2} \psi_{N-1}$. The eigenvalues of the edge states are $E= \pm i \sqrt{\gamma^{2}-g_{2}^{2}}$, implying that the large non-Hermiticity induces a pair of amplified and damped edge states with their frequency shifted to the waveguide resonant frequency. Correspondingly, the amplified edge state with energy $E=i \sqrt{\gamma^{2}-g_{2}^{2}}$ is

$$
\psi_{r}=\left[\ldots, 0, \rho_{-}^{2}, \rho_{-}, 0, \rho_{-}, 1\right]^{T}
$$

this state is located on the right boundary with decay factor $\rho_{-}=-i\left(\gamma-\sqrt{\gamma^{2}-g_{2}^{2}}\right) / g_{2}$. Conversely, the damped edge state with energy $E=-i \sqrt{\gamma^{2}-g_{2}^{2}}$ is

$$
\psi_{l}=\left[1, \rho_{+}, 0, \rho_{+}, \rho_{+}^{2}, 0, \ldots\right]^{T} ;
$$

this state is located on the left boundary with decay factor $\rho_{+}=+i\left(\gamma-\sqrt{\gamma^{2}-g_{2}^{2}}\right) / g_{2}$. The amplitude of the unit cell decays as $\rho_{ \pm}^{p}$ from the lattice boundary.

The edge states are localized at the lattice boundary and decay exponentially; therefore, we analytically obtain the confinements for the three configurations as the system size approaches infinity $(N \rightarrow \infty)$. Detailed calculations of the edge states are presented in the Appendix. For a system composed of integer unit cells $(N=3 n)$, the edge states exist when $g_{2}>g_{1}$ or the gain and loss satisfy

$$
\gamma>\gamma_{\mathrm{c}, \mathrm{e}}=g_{2}+g_{1}^{2} / g_{2}
$$

when $g_{2} \leqslant g_{1}$. The lattice with $N=3 n-1$ has two defects in the unit cell at each boundary. Edge states exist when the gain and loss rates are larger than the coupling $g_{2}$,

$$
\gamma>\gamma_{\mathrm{c}, \mathrm{e}}=g_{2}
$$

and for a system with $N=3 n-2$, which is a lattice with one missing site at each boundary of the unit cell, the edge states exist when $g_{1}>g_{2}$ or the gain and loss satisfy

$$
\gamma>\gamma_{\mathrm{c}, \mathrm{e}}=g_{2}+g_{1}^{2} / g_{2}
$$

when $g_{1} \leqslant g_{2}$. The threshold $\gamma_{\mathrm{c}, \mathrm{e}}$ is depicted in Fig. 5 for the three $\mathcal{P} \mathcal{T}$-symmetric configurations. As shown in Figs. 5(a) and 5(c), the $\gamma_{\mathrm{c}, \mathrm{e}}$ for finite systems of $n=30$ (red circles) is slightly larger than that predicted for infinite systems (black lines), whereas the $\gamma_{\mathrm{c}, \mathrm{e}}$ for a finite system of $n=300$ (blue squares) approaches that predicted for infinite systems. Notably, $\gamma_{\mathrm{c}, \mathrm{e}}$ in Fig. 5(b) is system-size independent; in this case, there is one pair of eigenstates $E^{2}=g_{2}^{2}-\gamma^{2}$ and the amplitudes at the passive sites (without gain or loss) vanish. Therefore, the wave functions are zero for every other pair of sites. The eigenvalues are real and the eigenstates are $\mathcal{P} \mathcal{T}$ symmetric when $\gamma \leqslant g_{2}$; however, when $\gamma>g_{2}$, this pair of eigenstates become a pair of edge states with purely imaginary eigenvalues. The edge states in the three $\mathcal{P} \mathcal{T}$-symmetric configurations for $g_{1}=1, g_{2}=1.5$, and $\gamma=2.5$ are plotted in Fig. 6. The edge state located on the left boundary in Fig. 6(a)
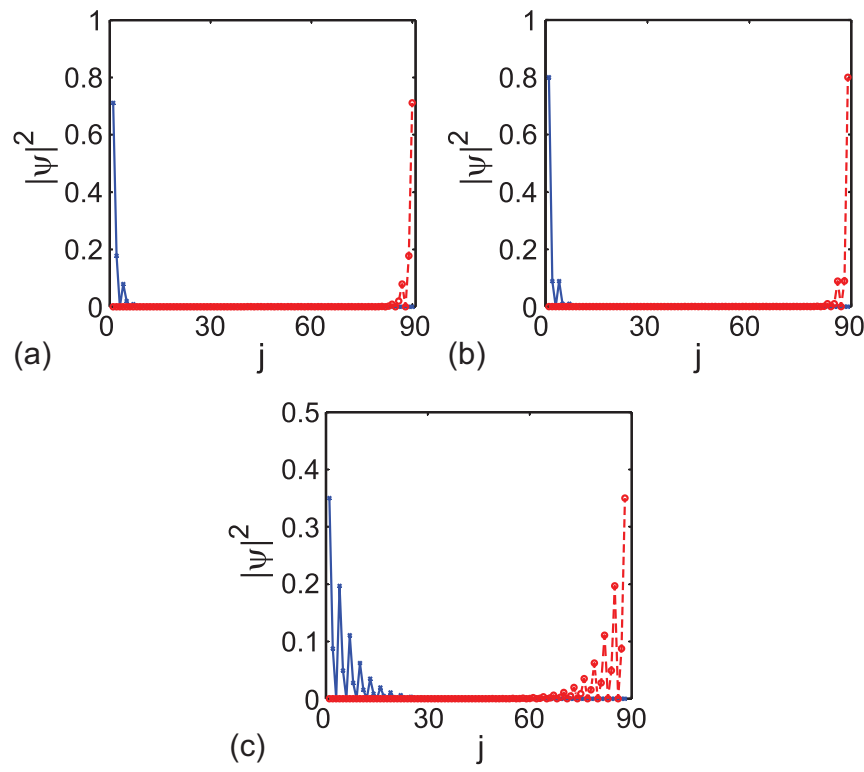

FIG. 6. Edge state probability distributions for $g_{2}=3 / 2$ and $\gamma=$ 2.5 for (a) $N=3 n=90$ with energies $\pm 2 i$, (b) $N=3 n-1=89$ with energies $\pm 2 i$, and (c) $N=3 n-2=88$ with energies $\pm i / 2$. 

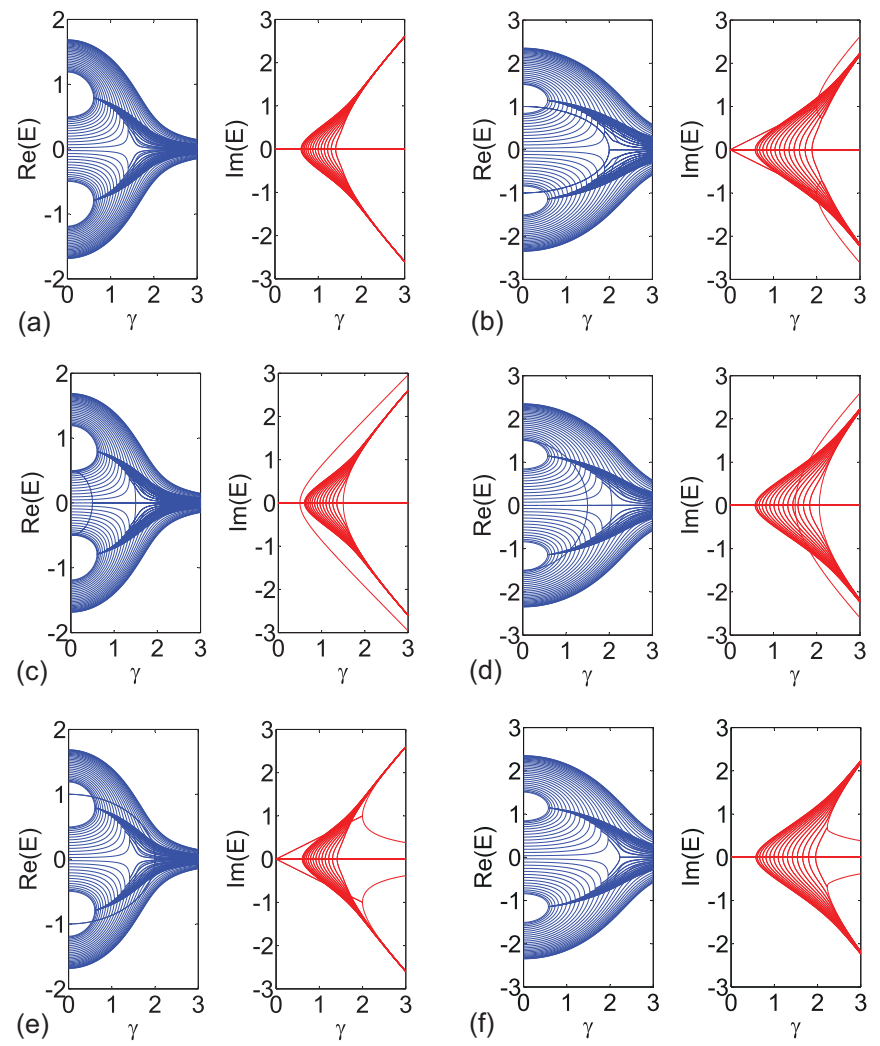

FIG. 7. Energy bands of $\mathcal{P} \mathcal{T}$-symmetric configurations as a function of $\gamma$ for (a) and (b) $N=3 n$, (c) and (d) $N=3 n-1$, and (e) and (f) $N=3 n-2$. The parameters are $g_{2}=1 / 2$ in the left plots and $g_{2}=3 / 2$ in the right plots. In all plots, $n=30$.

is the amplified mode, which is located on the right boundary in Figs. 6(b) and 6(c). These edge states are related to the edge state probability and gain (loss) distribution.

The spectra of $\mathcal{P} \mathcal{T}$-symmetric configurations are plotted as a function of $\gamma$ for $g_{1}=1$ and $g_{2}=1 / 2$ and 3/2 in Fig. 7 . As $\gamma$ is increased, the real parts of the spectra narrow, the band-gap widths are reduced, and amplification (attenuation) zero-mode edge states exist when the real parts are decreased to zero at $\gamma_{\mathrm{c}, \mathrm{e}}$, which is where the edge state frequencies are resonant with the waveguide lattice and their imaginary parts bifurcate. The thresholds $\gamma_{\mathrm{c}, \mathrm{e}}$ in Fig. 7 are consistent with the red circles in Fig. 5. In Fig. 7(a), $g_{1}>g_{2}$ and thus the threshold is dependent on the intercell coupling $g_{2}$, being $\gamma_{\mathrm{c}, \mathrm{e}}=2.655$; in Fig. 7(b), $g_{1}<g_{2}$, the threshold is $2 g_{1}$, being $\gamma_{\mathrm{c}, \mathrm{e}}=2.0$. Both of these cases are for a lattice size of $N=3 n=90$. The spectra presented in Figs. 7(c) and 7(d) are those for a lattice size $N=3 n-1=89$, where the threshold equals $g_{2}$, being $\gamma_{\mathrm{c}, \mathrm{e}}=0.5$ and 1.5 , respectively. For a lattice size $N=3 n-1$, the band gaps vanish when $g_{1}=g_{2}$. The bulk states at the top and bottom of the middle band become edge states when $\gamma>\gamma_{\mathrm{c}, \mathrm{e}}$ and $g_{1}>g_{2}$; the edge states are converted from the bulk states at the bottom of the top band and the top of the bottom band when $\gamma>\gamma_{\mathrm{c}, \mathrm{e}}$ and $g_{1}<g_{2}$. Figures 7(e) and 7(f) display the spectra obtained for a lattice size $N=3 n-2=88$ when $\gamma_{\mathrm{c}, \mathrm{e}}=2.0$ and $\gamma_{\mathrm{c}, \mathrm{e}}=2.226$, respectively. In Figs. 7(b) and $7(\mathrm{e})$, the edge states exist at any $\gamma$, have broken $\mathcal{P} \mathcal{T}$ symmetry, and have eigenvalues that linearly depend on $\gamma$ until bifurcation occurs at $\gamma=2.0$. Thereafter, zero-mode edge states exist and the amplification and attenuation of edge states depend on $\left(\gamma, g_{1}\right)$ under a square root function, as indicated by $E_{ \pm, \pm}$. In Figs. 7(a), 7(c), 7(d), and 7(f), no edge state exists when $\gamma<\gamma_{\mathrm{c}, \mathrm{e}}$ and a pair of bulk states becomes a conjugate pair of edge states when $\gamma>\gamma_{\mathrm{c}, \mathrm{e}}$. This pair of edge states is localized on opposite edges with identical frequency and an equal amount of amplification and attenuation.

\section{SUMMARY}

A topologically nontrivial non-Hermitian trimerized optical lattice is investigated. The $\mathcal{P} \mathcal{T}$-symmetric phases, band structure, and topologically protected edge states of a trimerized coupled waveguide lattice with universal non-Hermiticity are demonstrated. This lattice possesses topologies that are dependent on the degree of non-Hermiticity. In the topologically nontrivial region, the edge states are related to the configurations at the lattice boundary and depend on the coupling strengths. Two conjugate edge state pairs exist in the $\mathcal{P} \mathcal{T}$ symmetric configurations. These edge states are symmetrically arranged about the energy zero. Above a gain (loss) threshold, zero-mode edge states exist and these states are amplified and damped when propagating in opposite directions. Asymmetric transport through edge states in the photonic lattice represents a diode effect. The amplified edge state can be excited by dynamical creation, which is insensitive to the initial excitation or lattice imperfections. This robust one-way behavior has potential applications in optical manipulation, information processing, and unidirectional lasing.

\section{ACKNOWLEDGMENTS}

We acknowledge support from NSFC (Grant No. 11605094) and the Tianjin Natural Science Foundation (Grant No. 16JCYBJC40800).

\section{APPENDIX: SOLUTION OF THE EDGE STATES}

In the appendix we analyze the appearance of edge states in the three $\mathcal{P} \mathcal{T}$-symmetric configurations. For $N=3 n$, the Schrödinger equations of the edge states satisfy

$$
\begin{gathered}
(E-i \gamma) \psi_{1}=g_{1} \psi_{2}, \\
E \psi_{2}=g_{1} \psi_{1}, \\
0=g_{1} \psi_{2}+g_{2} \psi_{4},
\end{gathered}
$$

where $\psi_{j}$ is the wave function at waveguide $j$. We obtain $E^{2}-i \gamma E-g_{1}^{2}=0$. When $\gamma \leqslant 2 g_{1}$,

$$
\begin{gathered}
E_{ \pm}=\frac{i \gamma \pm \sqrt{4 g_{1}^{2}-\gamma^{2}}}{2} \\
\psi_{1}=-\frac{g_{2}}{\left(E_{ \pm}-i \gamma\right)} \psi_{4}
\end{gathered}
$$


The wave-function amplitude yields $\left|\psi_{1}\right|^{2} /\left|\psi_{4}\right|^{2}=g_{2}^{4} / g_{1}^{2}$ and the edge states exist when $g_{2}>g_{1}$. When $\gamma>2 g_{1}$,

$$
\begin{gathered}
E_{ \pm}=i \frac{\gamma \pm \sqrt{\gamma^{2}-4 g_{1}^{2}}}{2}, \\
\psi_{1}=\frac{2 g_{2}}{i\left(\gamma \mp \sqrt{\gamma^{2}-4 g_{1}^{2}}\right)} \psi_{4},
\end{gathered}
$$

and $\left|\psi_{1}\right|^{2} /\left|\psi_{4}\right|^{2}>1$ needs $4 g_{2}^{2}>\left(\gamma \mp \sqrt{\gamma^{2}-4 g_{1}^{2}}\right)^{2}$, that is, $2\left(g_{2}^{2}+g_{1}^{2}\right)-\gamma^{2}>\mp \gamma \sqrt{\gamma^{2}-4 g_{1}^{2}}$. If $\gamma^{2}<2\left(g_{2}^{2}+g_{1}^{2}\right)$, then the wave-function amplitude of $E_{+}$satisfies $\left|\psi_{1}\right|^{2} /\left|\psi_{4}\right|^{2}>1$; otherwise, $\gamma^{2}>2\left(g_{2}^{2}+g_{1}^{2}\right)$, the left side is less than zero, and $E_{-}$satisfies $\left|\psi_{1}\right|^{2} /\left|\psi_{4}\right|^{2}>1$, which requires $\gamma^{2}-2\left(g_{2}^{2}+\right.$ $\left.g_{1}^{2}\right)<\gamma \sqrt{\gamma^{2}-4 g_{1}^{2}}$, that is,

$$
\gamma>\left(g_{2}^{2}+g_{1}^{2}\right) / g_{2}
$$

Notably, $\gamma>2 g_{1}$ is already satisfied.

For $N=3 n-1$, the Schrödinger equations of the edge states satisfy

$$
\begin{gathered}
(E+i \gamma) \psi_{1}=g_{2} \psi_{2}, \\
(E-i \gamma) \psi_{2}=g_{2} \psi_{1}, \\
0=g_{1} \psi_{2}+g_{1} \psi_{4} .
\end{gathered}
$$

We obtain $E^{2}=g_{2}^{2}-\gamma^{2}, \quad \psi_{1}=-g_{2} \psi_{4}(E+i \gamma)$, and $\left|\psi_{1}\right|^{2} /\left|\psi_{4}\right|^{2}=1$ when $\gamma<g_{2}$. For $\gamma>g_{2}$, the energy $E$ becomes purely imaginary

$$
\begin{gathered}
E_{ \pm}= \pm i \sqrt{\gamma^{2}-g_{2}^{2}}, \\
\psi_{1}=-\frac{g_{2}}{i \gamma \pm i \sqrt{\gamma^{2}-g_{2}^{2}}} \psi_{4},
\end{gathered}
$$

$\left|\psi_{1}\right|^{2} /\left|\psi_{4}\right|^{2}=g_{2}^{2} /\left(\gamma \pm \sqrt{\gamma^{2}-g_{2}^{2}}\right)^{2}>1$ for $E_{-}=-i \sqrt{\gamma^{2}-g_{2}^{2}}$, and the corresponding edge state localizes on the left boundary. The edge state of zero real energy appears at $\gamma>g_{2}$ for lattice size $N=3 n-1$.

For $N=3 n-2$, the Schrödinger equations of the edge states satisfy

$$
\begin{gathered}
E \psi_{1}=g_{1} \psi_{2}, \\
(E+i \gamma) \psi_{2}=g_{1} \psi_{1}, \\
0=g_{2} \psi_{2}+g_{1} \psi_{4}
\end{gathered}
$$

and we obtain $E^{2}-i \gamma E-g_{1}^{2}=0$. When $\gamma \leqslant 2 g_{1}$,

$$
\begin{gathered}
E_{ \pm}=\frac{-i \gamma \pm \sqrt{4 g_{1}^{2}-\gamma^{2}}}{2}, \\
\psi_{1}=-\frac{g_{1}^{2} / g_{2}}{E_{ \pm}} \psi_{4},
\end{gathered}
$$

$\left|\psi_{1}\right|^{2} /\left|\psi_{4}\right|^{2}=g_{1}^{2} / g_{2}^{2}$, and the edge states exist when $g_{1}>g_{2}$. When $\gamma>2 g_{1}$,

$$
\begin{gathered}
E_{ \pm}=i \frac{-\gamma \pm \sqrt{\gamma^{2}-4 g_{1}^{2}}}{2} \\
\psi_{1}=-\frac{2 g_{1}^{2} / g_{2}}{i\left(-\gamma \pm \sqrt{\gamma^{2}-4 g_{1}^{2}}\right)} \psi_{4}
\end{gathered}
$$

and $\left|\psi_{1}\right|^{2} /\left|\psi_{4}\right|^{2}>1$ requires $4 g_{1}^{4} / g_{2}^{2}>\left(-\gamma \pm \sqrt{\gamma^{2}-4 g_{1}^{2}}\right)^{2}$, that is,

$$
\left(2 g_{1}^{4} / g_{2}^{2}+2 g_{1}^{2}-\gamma^{2}\right)>\mp \gamma \sqrt{\gamma^{2}-4 g_{1}^{2}} .
$$

If $2 g_{1}^{4} / g_{2}^{2}+2 g_{1}^{2}-\gamma^{2}>0$, that is, $\gamma^{2}<2 g_{1}^{4} / g_{2}^{2}+2 g_{1}^{2}$, then $E_{+}$satisfies $\left|\psi_{1}\right|^{2} /\left|\psi_{4}\right|^{2}>1$; otherwise, $2 g_{1}^{4} / g_{2}^{2}+2 g_{1}^{2}-$ $\gamma^{2}<0$, which requires $\gamma^{2}-2 g_{1}^{4} / g_{2}^{2}-2 g_{1}^{2}<\gamma \sqrt{\gamma^{2}-4 g_{1}^{2}}$, that is,

$$
\gamma>\left(g_{1}^{2}+g_{2}^{2}\right) / g_{2}
$$

[1] M. Z. Hasan and C. L. Kane, Rev. Mod. Phys. 82, 3045 (2010). [2] X.-L. Qi and S.-C. Zhang, Rev. Mod. Phys. 83, 1057 (2011).

[3] A. B. Khanikaev, S. H. Mousavi, W.-K. Tse, M. Kargarian, A. H. MacDonald, and G. Shvets, Nat. Mater. 12, 233 (2013).

[4] M. C. Rechtsman, J. M. Zeuner, Y. Plotnik, Y. Lumer, D. Podolsky, F. Dreisow, S. Nolte, M. Segev, and A. Szameit, Nature (London) 496, 196 (2013).

[5] L. Lu, J. D. Joannopoulos, and M. Soljačić, Nat. Photon. 8, 821 (2014).

[6] M. Hafezi, E. A. Demler, M. D. Lukin, and J. M. Taylor, Nat. Phys. 7, 907 (2011).

[7] N. Goldman, J. C. Budich, and P. Zoller, Nat. Phys. 12, 639 (2016).

[8] W.-P. Su, J. R. Schrieffer, and A. J. Heeger, Phys. Rev. Lett. 42, 1698 (1979).

[9] S. Ryu and Y. Hatsugai, Phys. Rev. Lett. 89, 077002 (2002).

[10] C. L. Kane and E. J. Mele, Phys. Rev. Lett. 95, 226801 (2005).
[11] B. A. Bernevig and S.-C. Zhang, Phys. Rev. Lett. 96, 106802 (2006).

[12] B. A. Bernevig, T. L. Hughes, and S. C. Zhang, Science 314, 1757 (2006).

[13] X.-L. Qi, Y.-S. Wu, and S.-C. Zhang, Phys. Rev. B 74, 085308 (2006).

[14] L. Fu, C. L. Kane, and E. J. Mele, Phys. Rev. Lett. 98, 106803 (2007).

[15] K. Esaki, M. Sato, M. Kohmoto, and B. I. Halperin, Phys. Rev. B 80, 125405 (2009).

[16] A. Y. Kitaev, in Advances in Theoretical Physics: Landau Memorial Conference, edited by V. Lebedev and M. Feigel'man, AIP Conf. Proc. No. 1134 (AIP, New York, 2009), p. 22.

[17] S. Ryu, A. Schneider, A. Furusaki, and A. Ludwig, New J. Phys. 12, 065010 (2010).

[18] L.-J. Lang, X. Cai, and S. Chen, Phys. Rev. Lett. 108, 220401 (2012). 
[19] H. M. Guo, Sci. China Phys. Mech. Astron. 59, 637401 (2016).

[20] H. Wang, Z. Ye, Y. Zhang, and N. Wang, Sci. Bull. 61, 1126 (2016).

[21] M. Atala, M. Aidelsburger, J. T. Barreiro, D. Abanin, T. Kitagawa, E. Demler, and I. Bloch, Nat. Phys. 9, 795 (2013).

[22] A. Ruschhaupt, F. Delgado, and J. G. Muga, J. Phys. A: Math. Gen. 38, L171 (2005).

[23] R. El-Ganainy, K. G. Makris, D. N. Christodoulides, and Z. H. Musslimani, Opt. Lett. 32, 2632 (2007).

[24] Z. H. Musslimani, K. G. Makris, R. El-Ganainy, and D. N. Christodoulides, Phys. Rev. Lett. 100, 030402 (2008).

[25] A. Guo, G. J. Salamo, D. Duchesne, R. Morandotti, M. VolatierRavat, V. Aimez, G. A. Siviloglou, and D. N. Christodoulides, Phys. Rev. Lett. 103, 093902 (2009).

[26] H. Jing, S. K. Özdemir, X. Y. Lü, J. Zhang, L. Yang, and F. Nori, Phys. Rev. Lett. 113, 053604 (2014).

[27] B. Peng, S. K. Özdemir, F. Lei, F. Monifi, M. Gianfreda, G. L. Long, S. Fan, F. Nori, C. M. Bender, and L. Yang, Nat. Phys. 10, 394 (2014).

[28] B. Peng, S. K. Özdemir, S. Rotter, H. Yilmaz, M. Liertzer, F. Monifi, C. M. Bender, F. Nori, and L. Yang, Science 346, 328 (2014).

[29] L. Chang, X. Jiang, S. Hua, C. Yang, J. Wen, L. Jiang, G. Li, G. Wang, and M. Xiao, Nat. Photon. 8, 524 (2014).

[30] H. Jing, S. K. Özdemir, Z. Geng, J. Zhang, X.-Y. Lü, B. Peng, L. Yang, and F. Nori, Sci. Rep. 5, 9663 (2015).

[31] X. Y. Lü, H. Jing, J. Y. Ma, and Y. Wu, Phys. Rev. Lett. 114, 253601 (2015).

[32] Z. P. Liu, J. Zhang, S. K. Özdemir, B. Peng, H. Jing, X. Y. Lü, C. W. Li, L. Yang, F. Nori, and Y. X. Liu, Phys. Rev. Lett. 117, 110802 (2016).

[33] C. Hang, G. Huang, and V. V. Konotop, Phys. Rev. Lett. 110, 083604 (2013).

[34] Z. Zhang, Y. Zhang, J. Sheng, L. Yang, M.-A. Miri, D. N. Christodoulides, B. He, Y. Zhang, and M. Xiao, Phys. Rev. Lett. 117, 123601 (2016).

[35] C. M. Bender and S. Boettcher, Phys. Rev. Lett. 80, 5243 (1998).

[36] P. Dorey, C. Dunning, and R. Tateo, J. Phys. A 34, 5679 (2001).

[37] A. Mostafazadeh, J. Math. Phys. 43, 205 (2002).

[38] H. F. Jones, J. Phys. A 38, 1741 (2005).

[39] S. Klaiman, U. Günther, and N. Moiseyev, Phys. Rev. Lett. 101, 080402 (2008).

[40] M. Znojil, Phys. Rev. D 78, 025026 (2008).

[41] L. Jin and Z. Song, Phys. Rev. A 80, 052107 (2009).

[42] I. Rotter, J. Phys. A: Math. Theor. 42, 153001 (2009).

[43] Y. N. Joglekar and J. L. Barnett, Phys. Rev. A 84, 024103 (2011).

[44] C. M. Bender, D. C. Brody, H. F. Jones, and B. K. Meister, Phys. Rev. Lett. 98, 040403 (2007).

[45] A. Mostafazadeh, Phys. Rev. Lett. 99, 130502 (2007).

[46] U. Günther and B. F. Samsonov, Phys. Rev. Lett. 101, 230404 (2008).
[47] C. Zheng, L. Hao, and G. L. Long, Philos. Trans. R. Soc. A 371, 20120053 (2013).

[48] C. E. Rüter, K. G. Makris, R. El-Ganainy, D. N. Christodoulides, M. Segev, and D. Kip, Nat. Phys. 6, 192 (2010).

[49] L. Feng, Y.-L. Xu, W. S. Fegadolli, M.-H. Lu, J. E. B. Oliveira, V. R. Almeida, Y.-F. Chen, and A. Scherer, Nat. Mater. 12, 108 (2013).

[50] Y. D. Chong, L. Ge, H. Cao, and A. D. Stone, Phys. Rev. Lett. 105, 053901 (2010).

[51] W. Wan, Y. Chong, L. Ge, H. Noh, A. D. Stone, and H. Cao, Science 331, 889 (2011).

[52] Y. Sun, W. Tan, H. Q. Li, J. Li, and H. Chen, Phys. Rev. Lett. 112, 143903 (2014).

[53] B. Zhu, R. Lü, and S. Chen, Phys. Rev. A 89, 062102 (2014).

[54] S. Lin, X. Z. Zhang, C. Li, and Z. Song, Phys. Rev. A 94, 042133 (2016).

[55] F. Ruzicka, Int. J. Theor. Phys. 54, 4154 (2015).

[56] C. Yuce, Phys. Lett. A 379, 1213 (2015).

[57] L. Jin, P. Wang, and Z. Song, Sci. Rep. 7, 5903 (2017).

[58] G. Q. Liang and Y. D. Chong, Phys. Rev. Lett. 110, 203904 (2013).

[59] J. M. Zeuner, M. C. Rechtsman, Y. Plotnik, Y. Lumer, S. Nolte, M. S. Rudner, M. Segev, and A. Szameit, Phys. Rev. Lett. 115, 040402 (2015).

[60] S. Malzard, C. Poli, and H. Schomerus, Phys. Rev. Lett. 115, 200402 (2015).

[61] C. Poli, M. Bellec, U. Kuhl, F. Mortessagne, and H. Schomerus, Nat. Commun. 6, 6710 (2015).

[62] D. Leykam, K. Y. Bliokh, C. Huang, Y. D. Chong, and F. Nori, Phys. Rev. Lett. 118, 040401 (2017).

[63] S. Weimann, M. Kremer, Y. Plotnik, Y. Lumer, S. Nolte, K. G. Makris, M. Segev, M. C. Rechtsman, and A. Szameit, Nat. Mater. 16, 433 (2017).

[64] H. Zhao, S. Longhi, and F. Feng, Sci. Rep. 5, 17022 (2015).

[65] K. Esaki, M. Sato, K. Hasebe, and M. Kohmoto, Phys. Rev. B 84, 205128 (2011).

[66] C. Yuce, Phys. Lett. A 378, 2024 (2014).

[67] A. K. Harter, T. E. Lee, and Y. N. Joglekar, Phys. Rev. A 93, 062101 (2016).

[68] X. Wang, T. Liu, Y. Xiong, and P. Tong, Phys. Rev. A 92, 012116 (2015).

[69] M. Klett, H. Cartarius, D. Dast, J. Main, and G. Wunner, Phys. Rev. A 95, 053626 (2017).

[70] Y. C. Hu and T. L. Hughes, Phys. Rev. B 84, 153101 (2011).

[71] S. Ganeshan, K. Sun, and S. Das Sarma, Phys. Rev. Lett. 110, 180403 (2013).

[72] C. H. Liang, D. D. Scott, and Y. N. Joglekar, Phys. Rev. A 89, 030102(R) (2014).

[73] J. Zak, Phys. Rev. Lett. 62, 2747 (1989).

[74] K. Ding, Z. Q. Zhang, and C. T. Chan, Phys. Rev. B 92, 235310 (2015). 